\title{
Syndrome Signature in Output Compaction for VLSI Built-in Self-Test
}

\author{
SUNIL R. DAS ${ }^{a, *, \dagger}$, NITA GOEL ${ }^{b}$, WEN B. JONE ${ }^{c}$ and AMIYA R. NAYAK \\ ${ }^{a}$ Presently with Dept. of Electrical Engineering and Computer Sciences, Computer Science Division, \\ University of California, Berkeley, CA 94720, U.S.A.; \\ ${ }^{\mathrm{b}}$ Dept. of Electrical Engineering, Faculty of Engineering, University of Ottawa, Ottawa, Ontario, Canada K1N 6N5; \\ ${ }^{c}$ Dept. of Computer Science and Information Engineering, National Chung Cheng University, Chiayi 62107, Taiwan, R.O.C.' \\ ${ }^{\mathrm{d} S c h o o l}$ of Computer Science, Carleton University, Ottawa, Ontario, Canada K1S $5 B 6$
}

(Received 10 June 1997)

\begin{abstract}
In this paper, we focus on the use of signature-based output compaction technique for built-in self-testing of VLSI circuits. We give algorithm for single-output and multipleoutput signature generation using exhaustive test patterns extending the syndrome concept. The signature we develop is a functional signature and is very effective for both input and internal line fault detection, as seen from simulation on various benchmark circuits. The signature generators can be easily implemented using the current VLSI technology.
\end{abstract}

Keywords: Syndrome signature, output compaction, built-in self-testing, VLSI circuits

\section{INTRODUCTION}

As the digital circuit technology is moving to high densities of integration, built-in self-testing (BIST) has become a primary issue in the realm of VLSI circuit design. Techniques for design for testability and built-in self-test consider the testing problem during the design stage of digital devices and have been found to be extremely effective. The central idea behind built-in self-testing or BIST is to have the chip test itself. This technique generates test patterns and evaluates output responses inside the chip $[4,9,12]$. It is already well recognized that
BIST can significantly improve the testability of VLSI chips and save testing time as well.

The test patterns used in BIST can be either random or exhaustive. Exhaustive test patterns have the following advantages over random test patterns:

- test patterns can be generated with relative ease, - no specific fault models are required, and - a high fault coverage can be achieved.

However, the main drawback of using exhaustive test patterns is the exponential growth of test length with increase in the number of inputs [8].

\footnotetext{
*(on sabbatical leave from Dept. of Electrical Engineering, Faculty of Engineering, University of Ottawa, Ottawa, Ontario, Canada K1N 6N5).

${ }^{\dagger}$ Corresponding author.
} 
The test output responses in BIST are compressed by output response analyzer into signature. The signature is compared with a known correct value, and faults are detected if a match does not occur. The various available output compaction techniques include parity-bit checking [1-3], transition count [6], ones count [11], Walsh coefficients $[7,13]$, and linear feedback shift register or LFSR [5]. Based on these approaches, the compressed response data can be used to evaluate the correctness of the circuit under test (CUT).

In this paper, we have proposed an output data compression technique called syndrome signature, particularly suited for exhaustive testing of VLSI circuits. The syndrome signature is based on and an extension of the novel idea of syndrome of a circuit originally conceived by Savir [10] and is related to the number of minterms realized by the corresponding switching function. The signature derived is an $(n+1)$-element vector consisting of the primary syndrome of the function and $n$ other subsyndromes corresponding to the subfunctions obtained by setting each of the $n$ input variables to 0 or 1 . The proposed technique is implemented on various ISCAS 85 benchmark combinational circuits (both single- and multiple-output), and the results look very encouraging.

\section{SYNDROME SIGNATURES}

A single-output syndrome signature is defined as follows. For an $n$-input single-output combinational switching function $F$ with input variables $x_{1}, x_{2}, \ldots, x_{n}$, the syndrome signature $s(F)$ of $F$ is given by an $(n+1)$-element vector $s(F)=$ $\left\langle s_{0}, s_{1}, \ldots, s_{n}\right\rangle$ where $s_{0}=s_{0}(F)$ and $s_{i}(F)=s_{i}\left(F_{0}^{i}\right)$, $i=1,2, \ldots, n, s_{0}$ denoting the primary syndrome, while $s_{i}$ denoting the subsyndrome of the subfunction $\left(F_{b}^{i}\right)$ obtained by setting the $i$ th variable in $F$ equal to $b(0$ or 1$)$.

Example 1 Assume a 4-input combinational function $F\left(x_{1}, x_{2}, x_{3}, x_{4}\right)$ defined by the truth table of Table I. The syndrome signature $s(F)$ of $F$ is given by the five-element vector: $s(F)=\langle 3 / 8,5 / 8$, $1 / 2,1 / 2,1 / 8\rangle$ where $s_{0}=s_{0}(F)=3 / 8$, and $s_{i}=$ $s_{i}\left(F_{0}^{i}\right), i=1,2, \ldots, 4, s_{0}$ denoting the primary syndrome of the function $F$, with $s_{i}$ representing the syndrome of the subfunction $\left(F_{b}^{i}\right)$ obtained by setting the $i$ th variable in $F$ equal to $b(0$ or 1$)$ as shown in Table I.

Figure 1 shows a straightforward implementation of this $(n+1)$-element signature in which the $(n+1)$ bit streams of interest are directed towards syndrome registers with equality checkers as an extension of the scheme as used for primary syndrome by Savir [10].

TABLE I Syndrome signature for a four-variable function

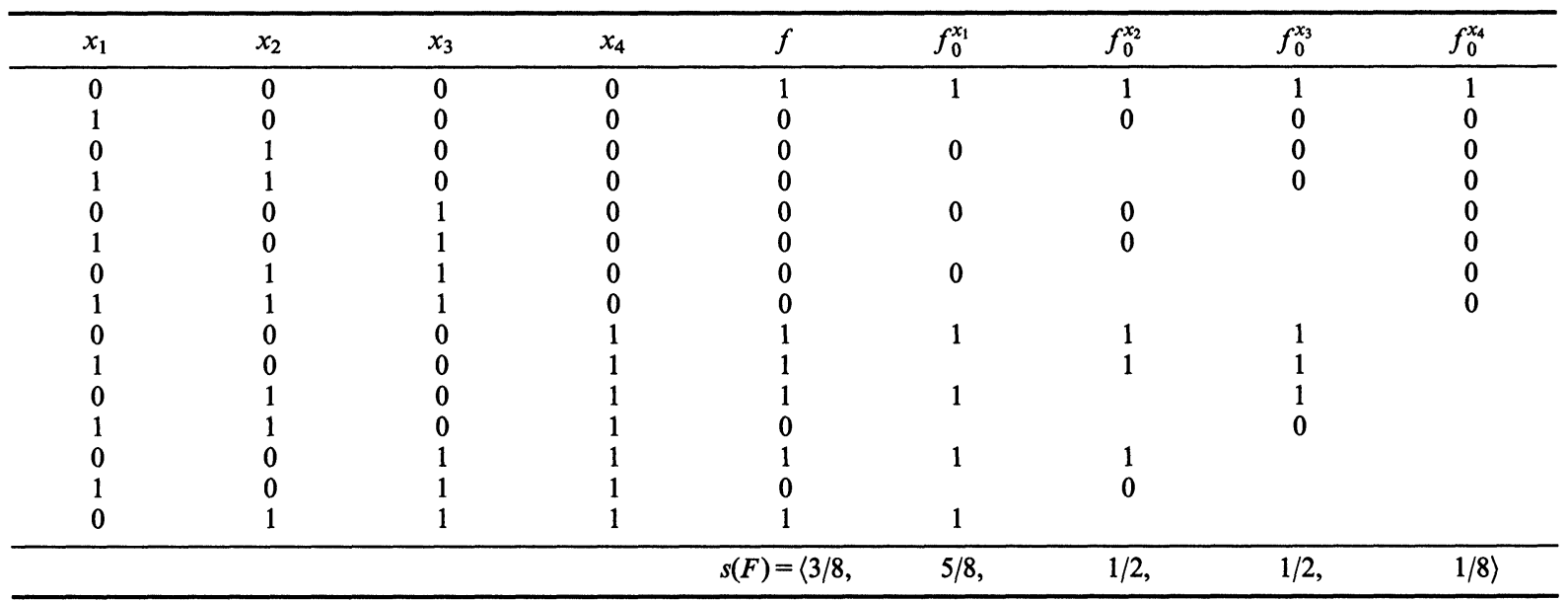




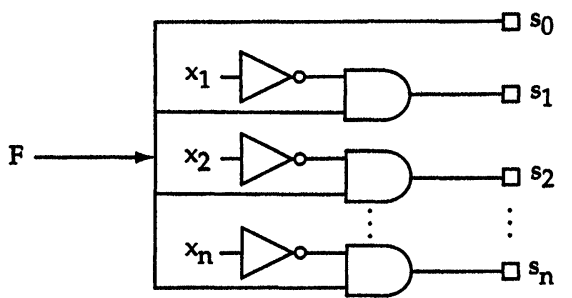

FIGURE 1 An implementation of single-output syndrome signature.

The concept of single-output syndrome signature can be readily extended to the multiple-output case. Evidently, the simplest strategy to extend the single-output syndrome signature to the multipleoutput case is to generate a separate signature for each output. Given an $n$-input $m$-output combinational circuit, a multiple-output syndrome signature is generated by EXORing all the outputs to produce a single new output which is then fed to a single-output syndrome signature generator.

Figure 2 shows the proposed implementation of a multiple-output syndrome signature. Consider as illustration of multiple-output syndrome signature the following example.

Example 2 A 4-input 2-output function together with its multiple-output syndrome signature is given in Table II.

\section{SYNDROME SIGNATURE PROPERTIES}

Some desirable properties of syndrome signature $s(F)=\left\langle s_{0}, s_{1}, \ldots, s_{n}\right\rangle$ for an $n$-variable switching function $F$ are summarized below:

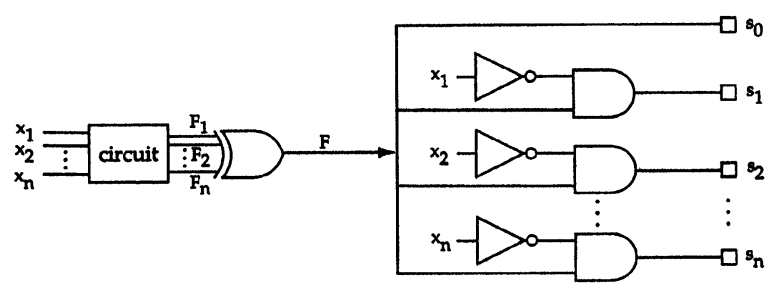

FIGURE 2 Proposed implementation of multiple-output syndrome signature.
THEOREM 1 A syndrome signature $s(F)=\left\langle s_{0}\right.$, $\left.s_{1}, \ldots, s_{n}\right\rangle$ for an $n$-variable switching function $F$ is a functional signature.

Proof The proof of the theorem follows obviously since a syndrome signature does not depend on the particular implementation involved in its realization.

Theorem $2 A$ syndrome signature $s(F)=\left\langle s_{0}\right.$, $\left.s_{1}, \ldots, s_{n}\right\rangle$ for an $n$-variable switching function $F$ is test-order independent.

Proof It is evident that the order in which the exhaustive test patterns are generated has no effect on the number of minterms realized by the function $F$ as well as by the $n$ subfunctions $s_{i}\left(F_{0}^{i}\right), i=$ $1,2, \ldots, n$, and hence in the signature.

Theorem 3 A syndrome signature $s(F)=\left\langle s_{0}\right.$, $\left.s_{1}, \ldots, s_{n}\right\rangle$ for an $n$-variable switching function $F$ is nonuniform.

Proof For an $n$-input arbitrary combinational function $F$, the parity-bit signature $s(F)$ of $F$ is uniform since all of the $2^{(n+1)}$ possible $(n+1)$-bit signatures are equally likely to result. With most counting procedures, this is, however, not the case. Some signatures are more likely to occur than others, thus making the signature nonuniform.

To prove the nonuniformity of the syndrome signature, consider the first element $s_{0}$ of the syndrome signature $s(F)$. Now $s_{0}$ is the syndrome of $F$ and can have any value (nonnormalized) between 0 and $2^{n}$.

Now there can be $2^{2^{n}}$ possible ways of assigning $2^{n}$ input combinations to $F$, and of these exactly ${ }^{2} C_{K}$ result in an $F$ with the same number of minterms or syndrome $K$. Hence, the probability that the function $F$ has the syndrome $K$ $\left(0 \leq K \leq 2^{n}\right)$ is ${ }^{n} C_{K} / 2^{2^{n}}$, which obviously is a function of $K$, unlike the probability of its parity $p(F)$ being 0 or 1 , which is $1 / 2$.

We can similarly prove this for the other elements of the signature $s(F)$. Consider, for instance, the $(i+1)$ th signature element $s_{i}, 0 \leq i \leq n$, 
TABLE II Multiple-output syndrome signature

\begin{tabular}{|c|c|c|c|c|c|c|c|c|c|c|}
\hline$x_{1}$ & $x_{2}$ & $x_{3}$ & $x_{4}$ & $f_{1}$ & $f_{2}$ & $f$ & $f_{0}^{x_{1}}$ & $f_{0}^{x_{2}}$ & $f_{0}^{x_{3}}$ & $f_{0}^{x_{4}}$ \\
\hline 0 & 0 & 0 & 0 & 1 & 0 & 1 & 1 & 1 & 1 & 1 \\
\hline 1 & 0 & 0 & 0 & 0 & 0 & 0 & & 0 & 0 & 0 \\
\hline 0 & 1 & 0 & 0 & 1 & 1 & 0 & 0 & & 0 & 0 \\
\hline 1 & 1 & 0 & 0 & 0 & 1 & 1 & & & 1 & 1 \\
\hline 0 & 0 & 1 & 0 & 0 & 0 & 0 & 0 & 0 & & 0 \\
\hline 1 & 0 & 1 & 0 & 1 & 0 & 1 & & 1 & & 1 \\
\hline 0 & 1 & 1 & 0 & 0 & 0 & 0 & 0 & & & 0 \\
\hline 1 & 1 & 1 & 0 & 0 & 0 & 0 & & & & 0 \\
\hline 0 & 0 & 0 & 1 & 1 & 0 & 1 & 1 & 1 & 1 & \\
\hline 1 & 0 & 0 & 1 & 1 & 1 & 0 & & 0 & 0 & \\
\hline 0 & 1 & 0 & 1 & 1 & 0 & 1 & 1 & & 1 & \\
\hline 1 & 1 & 0 & 1 & 0 & 0 & 0 & & & 0 & \\
\hline 0 & 0 & 1 & 1 & 1 & 0 & 1 & 1 & 1 & & \\
\hline 1 & 0 & 1 & 1 & 0 & 0 & 0 & & 0 & & \\
\hline 0 & 1 & 1 & 1 & 1 & 1 & 0 & 0 & & & \\
\hline \multirow[t]{2}{*}{1} & 1 & 1 & 1 & 0 & 1 & 1 & & & & \\
\hline & & & & & & & $1 / 2$ & $1 / 2$ & $1 / 2$ & $3 / 8\rangle$ \\
\hline
\end{tabular}

in $s(F)$. Now

$$
\begin{aligned}
s_{i} & =s\left(F^{x_{i}=0}\right) \\
& =s\left(\bar{x}_{i} \cdot F\right) \\
& =s\left[\bar{x}_{i} \cdot F\left(x_{1}, x_{2}, \ldots, x_{n}\right)\right] \\
& =s\left[F\left(x_{1}, x_{2}, \ldots, x_{i}=0, \ldots, x_{n}\right)\right] \\
& =s\left(F^{\prime}\right)
\end{aligned}
$$

where $F^{\prime}=\left(x_{1}, x_{2}, \ldots, x_{i}=0, \ldots, x_{n}\right)$ is a subfunction of $(n-1)$ variables $x_{1}, x_{2}, \ldots, x_{i-1}, x_{i+1}, \ldots, x_{n}$. Following identical reasoning as before, we can again show that this $(i+1)$ th element of $s(F)$ has a probability dependent on its syndrome value.

Thus, we conclude that all different $(n+1)$ elements of the signature can have any value (normalized) in the domain $\{0,1\}$, and therefore the signature is nonuniform.

The main advantage in using a syndrome signature rather than the primary syndrome is to achieve fault coverage of existing circuits without taking resort to a testable design which is impossible for off-the-shelf circuits. A fault which may not be syndrome testable could be syndrome signature testable. Now an important question that arises is: is the syndrome signature going to work for every case of single fault in a given circuit? The answer is obviously no, though it will work in most of the cases as our intensive experimentation with benchmark and other circuits conclusively shows.

THEOREM 4 Two $n$-variable switching functions $F_{1}$ and $F_{2}$ comprised of the same number of minterms $m$ and thus having the same syndrome $K=m / 2^{n}$ must have at least one subsyndrome corresponding to the subfunction $\left(F_{b}^{i}\right)$ obtained by setting the $i$ th variable in both $F_{1}$ and $F_{2}$ equal to $b$ $(0$ or 1$)$ different, i.e., $s_{i}\left(F_{10}^{i}\right) \neq s_{i}\left(F_{20}^{i}\right)$, in order that $F_{1}$ and $F_{2}$ are syndrome signature testable.

Proof The theorem follows from the very notion of syndrome signature testability.

COROLlary 1 If functions $F_{1}$ and $F_{2}$ are not syndrome signature testable because $s_{i}\left(F_{10}^{i}\right) \neq$ $s_{i}\left(F_{20}^{i}\right)$, then the implication is simply $N\left(\bar{x}_{i} F_{1}\right) \neq$ $N\left(\bar{x}_{i} F_{2}\right)$, where $N\left(\bar{x}_{i} F_{1}\right)$ and $N\left(\bar{x}_{i} F_{2}\right)$ denote respectively the number of minterms in the reduced functions $\bar{x}_{i} F_{1}$ and $\bar{x}_{i} F_{2}$.

THEOREM 5 Two n-variable switching functions $F_{1}$ and $F_{2}$ comprised of the same number of minterms $m$ and thus having the same syndrome $K=m / 2^{n}$ and $F_{1}=F_{2}$, may not have their syndrome signatures $s\left(F_{1}\right)$ and $s\left(F_{2}\right)$ different.

Proof We will prove the theorem by considering different situations in which $F_{1}$ and $F_{2}$ having the 
same number of minterms $m$, some of which are different.

Case $1 m-1$ minterms are identical, and only one minterm is different.

Obviously; since the functions consist of the same number of minterms, their primary syndromes are identical, i.e., $s_{0}\left(F_{1}\right)=s_{0}\left(F_{2}\right)$. Assume that the minterm $m_{1}$ of $F_{1}$ is different from the minterm $m_{2}$ of $F_{2}$. If we now examine the $n$-tuples corresponding to minterms $m_{1}$ and $m_{2}$, obviously $m_{1}$ and $m_{2}$ must differ in at least one variable position, say $x_{i}$. Here, $x_{i}$ is primed in one and unprimed in the other. Hence $N\left(\bar{x}_{i} F_{1}\right) \neq N\left(\bar{x}_{i} F_{2}\right)$, $N$ denoting the number of minterms in the respective reduced functions, according to Corollary 1. Thus, $s_{i}\left(F_{10}^{i}\right) \neq s_{i}\left(F_{20}^{i}\right)$, and the syndrome signatures are different.

Case $2 m-2$ terms are identical, and two minterms are different.

Assume $m_{1}, m_{2}$ of $F_{1}$ are different from $m_{1}^{\prime}, m_{2}^{\prime}$ of $F_{2}$. Then, $m_{1}, m_{2}$ and $m_{1}^{\prime}, m_{2}^{\prime}$ must differ in at least two variable positions, say $x_{i}$ and $x_{j}$. In this case, $N\left(\bar{x}_{i} F_{1}\right)=N\left(\bar{x}_{i} F_{2}\right)$ as well as $N\left(\bar{x}_{j} F_{1}\right)=$ $N\left(\bar{x}_{j} F_{2}\right)$, and hence $s_{i}\left(F_{10}^{i}\right)=s_{i}\left(F_{20}^{i}\right)$ and $s_{j}\left(F_{10}^{j}\right)=s_{j}\left(F_{20}^{j}\right)$.

Thus, there may be two switching functions comprised of the same number of minterms and having the same primary syndrome but might have some identical subsyndrome as well. This implies that their syndrome signatures are also identical.

Case 3 Similarly, we may consider the case where $F_{1}$ and $F_{2}$ are identical in $m-r$ minterms and differ in $r$ minterms, and can conclude that their syndrome signatures for the case could possibly be identical.

Thus, the theorem follows in general.

The following results are related and important in the context of syndrome signature testability.

TheOREM 6 Consider two n-variable switching functions $F_{1}$ and $F_{2}$ and let $F_{1}$ and $F_{2}$ differ in $M$ minterms. Then the minimum number of variables in whose assignments in the truth table these must differ equals

$$
\left\lceil\log _{2} M\right\rceil
$$

where $M=N_{1}+N_{2}, N_{1}$ and $N_{2}$ being the terms in $F_{1}$ and $F_{2}$ respectively which are different $\left(N_{1}=N_{2}=M / 2\right)$.

Corollary 2 Let $F_{1}$ and $F_{2}$ be two n-variable switching functions and let $F_{1}$ and $F_{2}$ have $M$ of their minterms different. Let $F_{1}$ and $F_{2}$ need $\left\lceil\log _{2} M\right\rceil$ variables with all their possible combinations in such a realization. If any variable $x_{i}$ has equal number of 0 's and 1's in M/2 minterms in $F_{1}$, any input line fault in $x_{i}$ cannot change $F_{1}$ to $F_{2}$ having the same distribution of 0's and 1's. That is, the distribution of 0 's and 1's must be different and as such

$$
N\left(\bar{x}_{i} F_{1}\right) \neq N\left(\bar{x}_{i} F_{2}\right)
$$

and

$$
s_{i}\left(F_{10}^{i}\right) \neq s_{i}\left(F_{20}^{i}\right)
$$

and hence the subsyndromes must be different for at least one such $x_{i}$.

\section{VLSI IMPLEMENTATION ISSUES}

The primary hardware in the implementation of the proposed signature generator is the syndrome register which can be readily implemented by using a counter. However, since the proposed signature generator, besides recording the primary syndrome, needs to record, for an $n$-variable function, $n$ other subsyndromes, $s_{i}\left(F_{0}^{i}\right)$, each corresponding to one of the variables, $x_{i}$, in a parallel implementation of the signature generator we need to use a total of $n+1$ counters, the first one counting from 0 to $2^{n}-1$, while the rest $n$ counters counting from 0 to $2^{n-1}-1$ each. This apparently leads to an excessive overhead in incorporating the signature 
generator in a self-test environment. This is obviously a drawback if the implementation has to be strictly a parallel implementation.

One simple way to overcome this obvious shortcoming of the proposed generator is to use a serial implementation. In the serial implementation a single counter can be used to record both the primary syndrome and the other subsyndromes. The amount of storage is the same although RAM is smaller than flip-flop. The test strategy can be appropriately modified to take into account the nature of implementation.

Finally, the recent advances in VLSI technology may not prove to be a deterrent even in the parallel implementation of the signature generator. The inclusion of only binary counters consistent with the number of primary inputs may not be big hardware overhead afterall, while compared with the complexity of the original circuit that needs to be tested using BIST.

\section{SYNDROME SIGNATURE ALGORITHM}

A procedure describing an algorithm for testing a circuit for syndrome signature testability is presented here. The algorithm first calculates the fault-free syndrome of the circuit and then calculates the faulty syndrome for any given fault in the circuit. If the two syndromes are different, the circuit is syndrome testable and the procedure stops; otherwise, it proceeds with the signature calculation and tests for syndrome signature testability.

\section{Procedure}

1. Given the number of inputs $n$, calculate the number of rows and the number of columns.

2. Given the faulty input and the fault in it, start the process of initialization:

$X(n)=0 \quad$ Fault-free input array

$X X(n)=0$ Faulty input array

$A_{\text {sum }}=0$ Sum of fault-free outputs, whenever the output is 1 . Final value of this will give the fault-free (actual) syndrome.

$F_{\text {sum }}=0$ Sum of faulty outputs, whenever the output is 1 . Final value of this will give the faulty syndrome.

and also, for $m=1, n$

$A_{\text {subs }}(m)=0 \quad$ Sum of the fault-free outputs for each of the reduced functions, whenever the output is 1 . Final value of this will give the actual subsyndromes.

$F_{\text {subs }}(m)=0$ Sum of the faulty outputs for each of the reduced faulty functions, whenever the output is 1 . Final value of this will give the faulty subsyndromes.

3. Main calculation loop

Generate next fault-free and faulty input pattern (to start with, an all 0's combination will be the first fault-free input pattern and an all 0's combination with a given fault inserted will be the corresponding faulty input pattern).

Calculate actual and faulty functions, $a_{F}$ and $f_{F}$ respectively and perform the following assignments:

If $a_{F}=1$, increment $A_{\text {sum }}$ by 1

If $f_{F}=1$, increment $F_{\text {sum }}$ by 1

Now calculate fault-free and faulty subsyndromes as:

For $m=1, n$

- If $X(m)=0$ and $a_{F}=1$ increase then, $A_{\text {subs }}(m)$ by 1

- If $X(m)=0$ and $f_{F}=1$ increase then, $F_{\text {subs }}(m)$ by 1

Repeat Step 3 until all the combinations ( $2 n)$ are done.

4. The decision to be made here is:

Is $A_{\text {sum }}$ equal to $F_{\text {sum }}$ ?

If false, then the circuit is syndrome testable and goto Step 2;

If true, try syndrome signature testing and goto Step 2 until all faults of interest are analyzed. 
Note Division by $2^{n}$ on final $A_{\text {sum }}$ and $F_{\text {sum }}$ and by $2^{n-1}$ on final $A_{\text {subs }}(m)$ and $F_{\text {subs }}(m)$ are not performed here (according to the definitions of syndrome and subsyndromes), as we are doing only the comparison. Also, this avoids the roundoff errors while computing these values as the circuit size increases.

\section{EXPERIMENTAL RESULTS}

To verify the proposed syndrome signature method, simulations were performed on combinational circuits. The simulator (implemented on a 486 PC) consists of an input pattern generator, logic simulator, output compressor, and fault generator. All possible single stuck-at faults were introduced on primary input lines and on internal lines of the ISCAS 85 benchmark circuits, and the outputs were compressed by syndrome signature technique. Fault-free signatures were compared with the faulty signatures using syndrome counters.

In general, the dominating factors in the simulation were the number of input patterns and the number of faults that were injected. Some of the benchmark circuits are not very large and can be handled quite easily whereas some others are really large with respect to the number of primary inputs, internal lines and primary outputs. Since our method of testing using syndrome signature (in case the primary syndrome fails) is exhaustive, as far as input test patterns are concerned, storage and time become a problem if the circuit has more than 20 inputs. One obvious way out of such a situation is to partition the circuit into certain subcircuits depending on the dependence of a particular output on a proper subset of the inputs to make the problem tractable. We used this technique in the case of large benchmark circuits.

The results obtained can be considered to be sufficient to show the implementation of the proposed technique on large combinational logic circuits. We have considered both, the primary input as well as the internal line faults. Since our major objective was only to see how the benchmark circuits relate to our concept of syndrome signature for detection of single faults, no attempt has been made to keep track of the CPU time.

It can be seen from the results that benchmark circuits "C17", "CKT" and a subcircuit (considering only that output which depends on less than 20 inputs) of "C432" are syndrome testable for both input and internal line faults. The fourth circuit, "C880", was partitioned into several subcircuits. Only two subcircuits of "C880" were considered for experimentation, one with 13 inputs and 9 outputs and the other with 10 inputs and 1 output. For some input and internal line faults, these subcircuits are syndrome testable, while for some they are syndrome signature testable. There are some faults which are neither syndrome nor syndrome signature testable.

Tables III and IV show the number of faults missed by syndrome (and their percentage) as well as the number of faults missed by syndrome signature (and their percentage) with respect to the total number of faults injected in each case of single-output and multiple-output circuits. In our simulation results, in case of single-output circuits, syndrome signature has provided the same fault coverage as that of syndrome, but for multipleoutput circuits syndrome signature has given better fault coverage than syndrome.

Since in most of the multiple-output benchmark circuits we used circuit partitioning, we never had to consider number of inputs exceeding 18 which generates $2^{18}$ test patterns and could be handled without appreciable difficulty. With today's computing power, normally $2^{20}$ test patterns could be generated in less than $1 \mathrm{~ms}$ and as such testing a particular fault of interest should not be much of a problem, even if the syndrome signature (which uses exhaustive test patterns) needs to be used. Obviously, the signature would be working in most of the cases where the syndrome fails, though there may be cases where this signature might also fail. 
TABLE III Single-output circuits

\begin{tabular}{lccccc}
\hline CUT & $N_{\text {Tot }}$ & $M_{s}$ & $\% M_{s}$ & $M_{s s}$ & none \\
\hline CKT & 24 & none & 0 & 0 \\
C432 & 72 & none & 0 & none & 54 \\
C880 & 84 & 54 & 64 & 64 \\
(10 inputs) & & & & \\
\hline
\end{tabular}

$N_{\text {Tot }}$ : Total number of faults injected.

$M_{s}$ : Number of faults missed by syndrome.

$M_{s s}$ : Number of faults missed by syndrome signature.

TABLE IV Multiple-output circuits

\begin{tabular}{lccccc}
\hline CUT & $N_{\text {Tot }}$ & $M_{s}$ & $\% M_{s}$ & $M_{s s}$ & $\% M_{s s}$ \\
\hline C17 & 18 & none & 0 & none & 0 \\
C880 & 38 & 6 & 16 & none & 0 \\
(13 inputs) & & & & & \\
\hline
\end{tabular}

$N_{\text {Tot }}$ : Total number of faults injected.

$M_{s}:$ Number of faults missed by syndrome.

$M_{s s}$ : Number of faults missed by syndrome signature.

\section{CONCLUSIONS}

In this paper, we have proposed an output data compression technique called syndrome signature, particularly suited for exhaustive testing of VLSI circuits. The syndrome signature is based on and an extension of the novel idea of syndrome of a circuit originally suggested by Savir [10] and is related to the number of minterms realized by the corresponding switching function. The results of implementation on some ISCAS 85 benchmark circuits (involving both single-output and multiple-output circuits) look very promising. Some circuits which according to Savir need extra inputs in order to make them syndrome testable, are shown to be syndrome signature testable. Also, the signature generation discussed for multiple-output circuits seems to work and preserves all the desirable properties of the single-output response analyzer. Masking effect of the proposed signature generator does not seem to be very high as only one case was reported from 11 sample circuits, use although an extensive simulation is needed for this statement to hold true, which is an important measure of a good compression technique.

Though this paper is not primarily about the VLSI implementation of the proposed signature generator, some thought has been given in this direction. The size of the compactor seems high as compared to the commonly used methods. The counters will require $O\left(n^{2}\right)$ flip-flops and $O\left(n^{2}\right)$ XORs and ANDs. In an $n$-input combinational circuit, this seems an excessive overhead in incorporating the signature generator in BIST environment. If the implementation is strictly a parallel implementation, this can be considered as a drawback of the proposed signature generator. On the other hand, the serial implementation has the advantage of requiring only $n$ flip-flops, but, a disadvantage of requiring $n * 2^{n}$ tests (thus increasing the test time). The number of memory cells required is the same as that of in parallel implementation.

As the main drawback of exhaustive test patterns is their exponential growth with the number of inputs, such exhaustive testing is acceptable in cases (most of the benchmark circuits) where combinational circuits can be partitioned into circuits of under 20 inputs to keep the testing time under control.

Further research in this area needs to focus on relative effectiveness of the syndrome signature technique as a useful test compaction tool for fault detection through implementation of many more 
real world circuits where conventional syndromes fail. Also, implementation of such a signature in VLSI could be a real worthwhile sequel to the whole exercise.

\section{Acknowledgement}

This research was supported in part by the Natural Sciences and Engineering Research Council of Canada under Grant A 4750. Portions of the results reported in the research were presented at the Ninth VLSI Design Conference held in Bangalore, India during January 3-6, 1996.

\section{References}

[1] Akers, S. B. (Mar. 1988). "A parity bit signature for exhaustive testing", IEEE Trans. Computer-Aided Design of VLSI Circuits and Systems, 7, pp. 333-338.

[2] Bhattacharya, B. B. and Seth, S. C. (Nov. 1989). "Design of parity testable combinational circuits", IEEE Trans. Computers, C-38, pp. 1580-1584.

[3] Carter, W. C. (Nov. 1982). "Signature testing with guaranteed bounds for fault coverage", Digest of Papers of Int. Test Conf., pp. 75-82.

[4] Das, S. R. (Oct. 1991). "Built-in self-testing of VLSI circuits", IEEE Potentials, 10, pp. 23-26.

[5] Frowerk, R. A. (May 1977). "Signature analysis - a new field service method", Hewlett-Packard Journal, 28, pp. 2-8.

[6] Hayes, J. P. (June 1976). "Transition count testing of combinational logic circuits", IEEE Trans. Computers, C-25, pp. 613-620.

[7] Hsiao, T.-C. and Seth, S. C. (Oct. 1984). "An analysis of the use of Rademacher-Walsh spectrum in compact testing”, IEEE Trans. Computers, C-33, pp. 934-937.

[8] Jone, W. B. and Das, S. R. (June 1990). "Multiple-output parity bit signature for exhaustive testing", Journal of Electronic Testing: Theory and Applications, 1, pp. 175178.

[9] McCluskey, E. J. (April 1985). "Built-in self-test techniques", IEEE Design and Test of Computers, 2, pp. 21-28.

[10] Savir, J. (June 1980). "Syndrome testable design of combinational circuits", IEEE Trans. Computers, C-29, pp. $442-451$.

[11] Savir, J. and McAuney, W. H. (1985). "On the masking probability with one's count and transition count", Proc. of Int. Conf. on Computer-Aided Design, pp. 111-113.

[12] Savir, J. and Bardell, P. H. (March 1993). "Built-in self-test: milestones and challenges", VLSI Design, 1, pp. 23-44.

[13] Susskind, A. K. (Feb. 1983). "Testing by verifying Walsh coefficients", IEEE Trans. Computers, C-32, pp. 198-201.

\section{Authors' Biographies}

Sunil R. Das is a Professor of Electrical and Computer Engineering at the School of Information Technology and Engineering, University of Ottawa, Ottawa, Ontario, Canada. He holds a B.Sc. (Honors) in Physics and an M.Sc. (Tech) and a Ph.D. in Radiophysics and Electronics from the University of Calcutta, Calcutta, West Bengal, India. He previously held academic positions with the Center for Reliable Computing (CRC), Computer Systems Laboratory, Department of Electrical Engineering, Stanford University, Stanford, CA (on sabbatical leave), the Institute of Computer Engineering, National Chiao Tung University, Hsinchu, Taiwan, Republic of China, and the Center of Advanced Study (CAS), Institute of Radiophysics and Electronics, University of Calcutta.

Dr. Das published extensively in the areas of switching and automata theory, digital logic design, threshold logic, fault-tolerant computing, microprogramming and microarchitecture, microcode optimization, applied theory of graphs, and combinatorics. He was elected one of the delegates of the prestigious Good People, Good Deeds of the Republic of China in 1981 in recognition of his outstanding contributions in the field of research and education.

Dr. Das is currently the Managing Editor of the IEEE VLSI Technical Bulletin, a publication of the IEEE Computer Society Technical Committee (TC) on VLSI, an Executive Committee Member of the IEEE Computer Society Technical Committee (TC) on VLSI, an Associate Editor of the IEEE Transactions on Systems, Man, and Cybernetics, an Associate Editor of the IEEE Transactions on VLSI Systems, and a Member of the Editorial Board and a Regional Editor for Canada of the VLSI Design: An International Journal of Custom-Chip Design, Simulation and Testing published by Gordon and Breach Science Publishers, Inc., NY. 
Dr. Das edited jointly with P. K. Srimani a book entitled, Distributed Mutual Exclusion Algorithms, published by the IEEE Computer Society Press, Los Alamitos, CA 1992 in its Technology Series. He is also the author jointly with C. L. Sheng of a text on Digital Logic Design being published by Ablex Publishing Corporation, Norwood, NJ. Dr. Das serves as the Co-Chair of the IEEE Computer Society Students Activities Committee from Region 7 (Canada).

Dr. Das is a Fellow of the Institute of Electrical and Electronics Engineers (IEEE), Inc. (with Membership in the IEEE Computer Society, IEEE Systems, Man, and Cybernetics Society, and IEEE Circuits and Systems Society), and a Member of the Association for Computing Machinery (ACM), U.S.A. He was elected a Fellow of the IEEE in 1994 for contributions to switching theory and computer design.

Dr. Das is the 1996 recipient of the IEEE Computer Society's highly esteemed Technical Achievement Award for his pioneering contributions in the fields of switching theory and modern digital design, digital circuits testing, microarchitecture and microprogram optimization, and combinatorics and graph theory.

On sabbatical leave from the University of Ottawa, Dr. Das is presently with the Department of Electrical Engineering and Computer Sciences, Computer Science Division, University of California, Berkeley, CA.

Nita Goel received the Bachelor's degree in Electronics and Telecommunications in 1985 from MBM Engineering College, Jodhpur, India and the Master's degree in Electrical Engineering in 1994 from the University of Ottawa, Canada. Since 1994, she is working for Tecsis Corporation where she is developing software applications for software assurance, databases for medical applications and web based tools and applications. Her interests are in developing applications for large software systems.

Wen-Ben Jone was born in Taipei, Taiwan, Republic of China. He received the B. S. degree in computer science in 1979 , the M. E. degree in computer engineering in 1981, both from National Chiao-Tung University, Taiwan; and the Ph.D. degree in computer engineering and science from Case Western Reserve University, Cleveland, $\mathrm{OH}$, in 1987.

In 1987, he joined the Department of Computer Science at New Mexico Institute of Mining and Technology, Socorro, where he was promoted as an associate professor in 1992 . He has been with the Department of Computer Engineering and Information Science, National Chung-Cheng University, Chiayi, Taiwan, R.O.C since 1993, and is currently a full professor. $\mathrm{He}$ was a visiting research fellow with the Department of Computer Science and Engineering, the Chinese University of Hong-Kong, in 1997 summer.

His research interests include fault-tolerant computing, VLSI design and test, and computer architecture. He has published more than 60 papers and served as a reviewer in these research areas in various technical journals and conferences. Prof. Jone is also listed in the Marquis Who's Who in the World (15th Edition, 1998). He has also served on the program committees of VLSI Design/CAD Symposium (since 1993, in Taiwan), 1995, 1996 Asian Test Conference, 1995-1997 Asia and South Pacific Design Automation Conference.

Dr. Jone received the Best Thesis Award from The Chinese Institute of Electrical Engineering, (Republic of China), in 1981. He is a member of IEEE and the IEEE Computer Society Test Technology Technical Committee.

Dr. Amiya R. Nayak got his B. Math in Computer Science from the University of Waterloo, Waterloo, Ontario, Canada in 1982, and his M.Sc. and Ph.D., also in Computer Science, from the Carleton University, Ottawa, Ontario in 1986 and 1991, respectively. Dr. Nayak worked for the Canadian Marconi Company in Kanata, Ontario for about 12 years before he moved to NORTEL in Ottawa as a technical consultant.

Dr. Nayak is an Adjunct Professor in the School of Computer Science at Carleton University. $\mathrm{He}$ is 
the Canadian Editor in charge of book reviews for VLSI Design published by Gordon and Breach Science Publishers, Inc., NY. Dr. Nayak published numerous articles in the areas of fault-tolerant computing, VLSI testing, and parallel and distributed computing.

Dr. Nayak is a Member of the IEEE and ACM, U.S.A. 

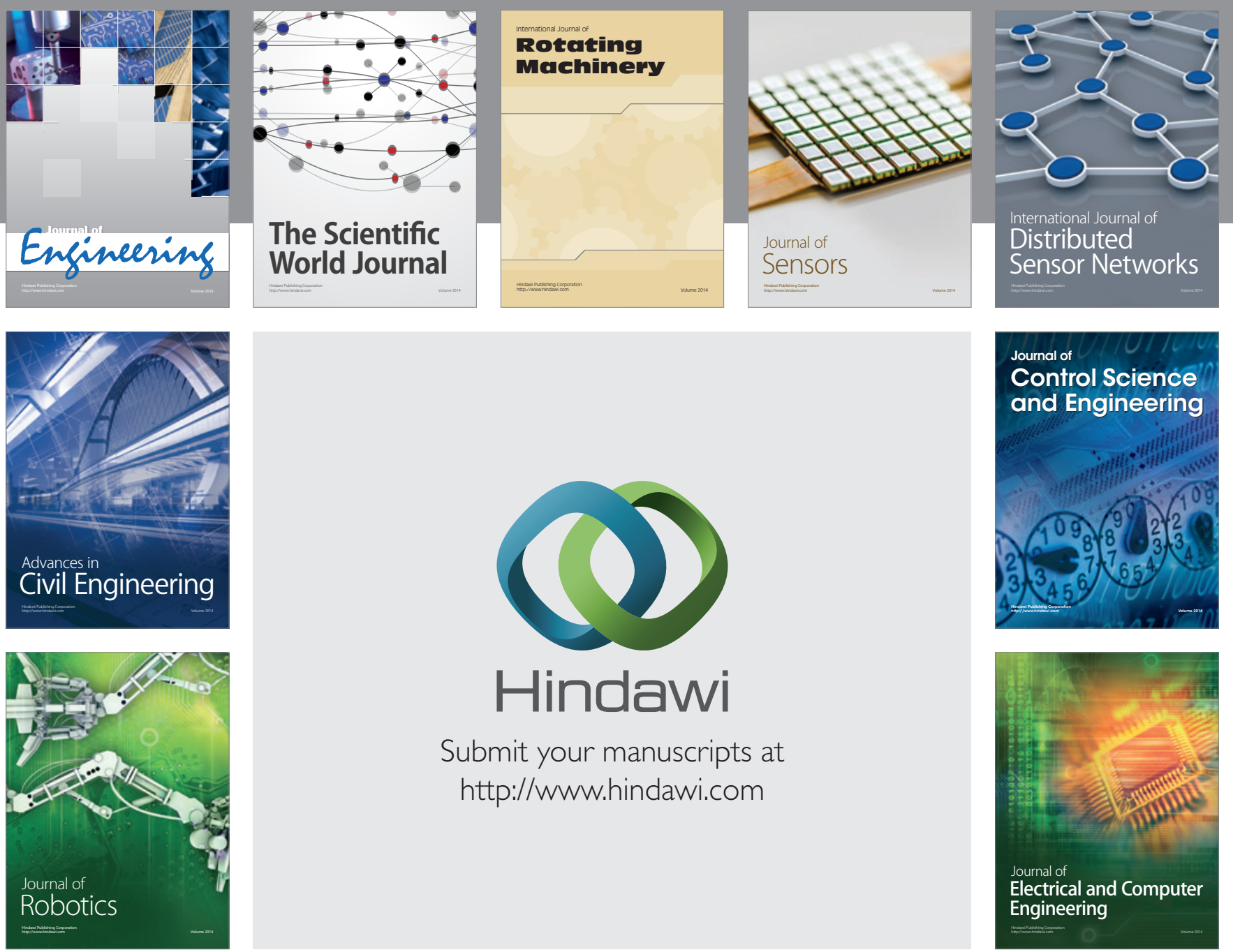

Submit your manuscripts at

http://www.hindawi.com
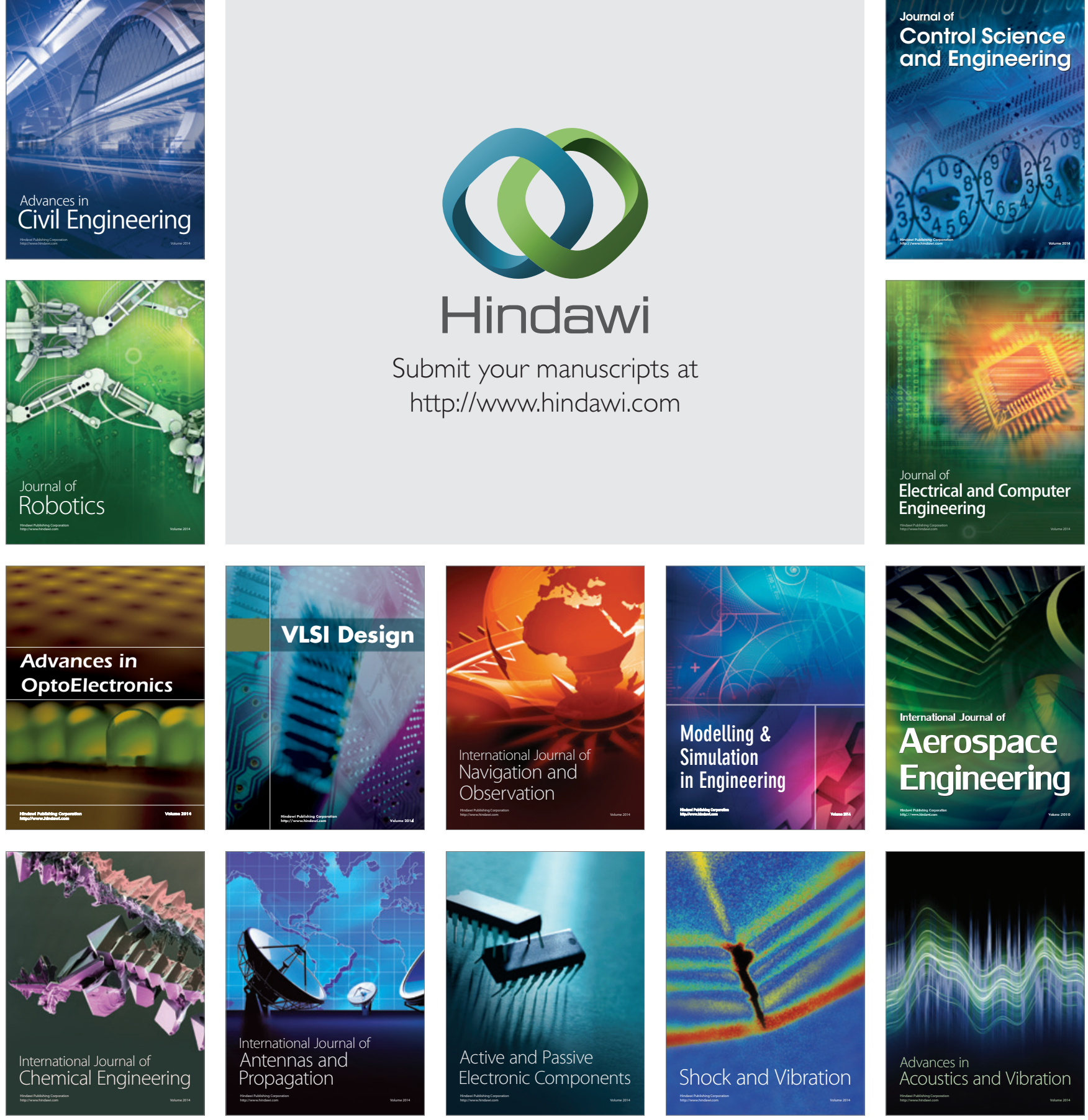